1997 Particle Accelerator Conf., Vancouver, B.C., Canada, May 12-16, 1997.

\title{
A Multi-wire Beam Profile Monitor in the AGS
}

\author{
H. Huang , W. Buxton, V. Castillo, J.W.Glenn, \\ G. Mahler, M. Syphers, W. van Asselt, R.L. Witkover, S.Y. Zhang \\ Brookhaven National Laboratory, Upton, NY 11973, USA
}

RECEIVED

JUN 251997

\begin{abstract}
A multi-wire beam profile monitor which can be used to directly monitor and control the optical matching between the Booster and AGS rings has been installed and tested in the AGS. Placement of a multi-wire monitor directly in the AGS provides profile measurements taken upon injection and the first two or more revolutions of the beam. The data from such measurements can be used to determine the optical properties of the beam transport line leading into the AGS.
\end{abstract}

\section{INTRODUCTION}

As the AGS prepares to serve as the injector for RHIC, monitoring and control of the beam transverse emittances will become a major and important topic. Before installing the multi-wire beam profile monitor, there was no device for directly measuring the optical matching between the Booster and AGS rings. Previously the only beam profile measurement device in the AGS was the Ionization Profile Monitor(IPM) [1]. However, the IPM measurement is integrated over a finite time interval and therefore can provide only the average profile over a great many number of turns. Hence, at injection, the profile obtained with the IPM is the profile after dilution has occured, instead of the profile as delivered by the Booster and BTA (Booster to AGS) beamline. Placement of a multi-wire monitor in the AGS can provide profile measurements taken upon injection and the first two or more revolutions of the beam. The data from such measurements can be used to determine the optical properties of the beam transport line leading into the AGS.

\section{HARDWARE AND ELECTRONICS}

Multi-wire profile monitors (harps) have been used in the HITL (Heavy Ion Transfer Line) [2], the ITB (Linac to Booster) line and the BTA line [3]. These are commercially produced devices which have proven to be very reliable. The newly installed harp built for the AGS is similar to existing units and is located in the A20 straight section which is close to the injection into the AGS.

Fig. 1 shows the arrangement of the harp wires. Horizontal beam-profile information is derived from the 64 wires that run vertically and the vertical beam-profile information is derived from the 32 wires that run horizontally. The $0.1 \mathrm{~mm}$ diameter tungsten wires are separated by $2 \mathrm{~mm}$ spacing to cover an area of $13.2 \mathrm{~cm} \times 6.4 \mathrm{~cm}$. The horizontal and vertical wires are mounted on the same plane. The harp is pneumatically inserted to a fixed position in the

\footnotetext{
- Work performed under the auspices of the U.S. Dept. of Energy
}

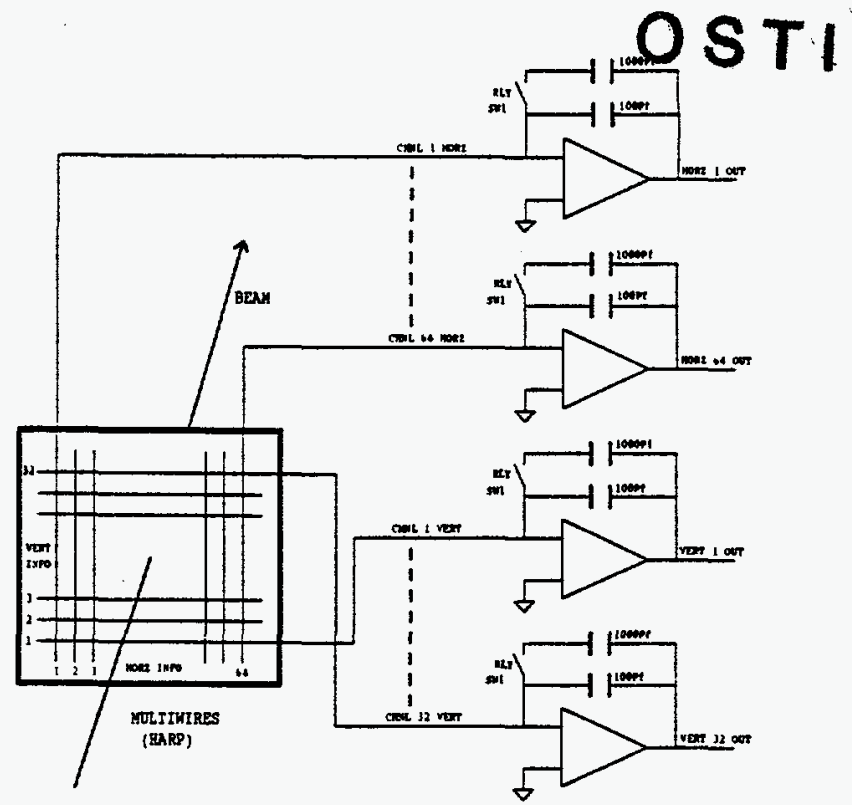

Figure 1: The A20 harp wires and corresponding integrator channels. The Reset, Integrate, and Hold switches are not shown for simplicity.

beam pipe. To insert, a 3-way solenoid valve on the harp is powered allowing 60 psi air to move a plunger which, in turn, moves the harp into the beam path; to extract, power is taken off the solenoid and the air moves the plunger in the opposite direction (out of the beam path). Microswitches close when the plunger reaches the end of its travel and readbacks indicating position are relayed to the control system.

In the A20 harp electronic system, charge from the multi-wires goes to individual gated integrator channels producing an output voltage. These voltages, 64 for horizontal information and 32 for vertical, go to $96 \mathrm{MUX}$ (sample and hold) channels which are read out sequentially, and fed to the control system which produces both transverse beam profiles displayed in the AGS main control room.

There are two main processes going on: the integration process during which the charge is collected from the wires in the beam path and the integrating capacitor is charged to a proportional voltage which is directly coupled to a MUX channel; and the scan process during which the voltages on the 96 MUX channels are sequentially read out to the control system.

When the A20 harp is inserted in the beam path the charge on the wires bleed off to the integrating capacitor. The integrating op-amp presents a high impedance input and in balancing the charge on the integrating capacitor 

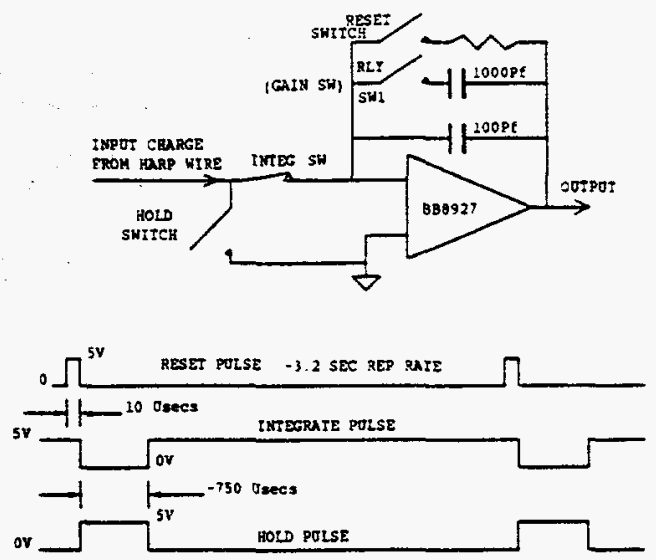

Figure 2: a) Integrator channel showing Reset, Integrate and Hold switches during the integration process. b) Timing signals that control the Reset, Integrate and Hold switches.

produces an output voltage. There are two available capacitor values: $100 \mathrm{pF}$ used as a high sensitivity range and $1100 \mathrm{pF}$, obtained by remotely closing the relay, used as a low sensitivity range.

The integration process is regulated by the Reset, Start Integrate and Hold (Stop integrate) timing pulses. An integrate channel is shown in Fig. 2 with the switches (FETs) that allow the regulation process. Also shown in Fig. 2 is the timing arrangement that controls these switches. The cycle starts with the Reset pulse shorting the integrating capacitor and thus zeroing the output voltage. Next, incoming Integrate and Hold pulses simultaneously close the Integrator switch and open the Hold switch allowing charge to get to the op-amp. After the integrate time, the process is reversed: the Hold switch closes and the Integrate switch opens simultaneously, shorting the incoming charge to ground. At this point, the integrator channels all have voltages on their outputs thus marking the end of the integrate process. These voltages are directly coupled to their respective MUX channels and the read-out process commences. To read out the MUX channels sequentially, each MUX chip is selected and its 16 channels are sequentially selected for readout by a group of 16 channel advance pulses. The sequence is continued for the 64 channels in the horizontal plane and 32 channels in the vertical plane, to complete the readout process and end the cycle. The next reset signal shorts the integrating capacitor, thus zeroing the output voltage and the cycle proceeds with incoming integrate and hold pulses.

\section{TESTS WITH HEAVY ION BEAM}

In determining the optical set-up of the beam transport system, it is relatively straightforward to measure the trajectory of the beam at the injection to the AGS and to measure how well it is matched to the closed orbit. This is



Figure 3: Vertical beam profile taken consecutively in the first three turns with the A20 harp. The lines are Gaussian fits. High sensitivity gain was used.

performed by looking at a beam position monitor to see the residual betatron oscillation after injection. A similarly straightforward process is a measurement of the dispersion function being delivered by the beamline. This can be performed by looking at motion on position monitors as the energy of the Booster is varied slightly. Previously, the difficult measurement in the AGS was the amplitude function being delivered by the beamline. The multi-wire profile monitor performs this measurement in the AGS.

A series of tests with Gold beam were conducted during the last heavy ion run. The tests have demonstrated that the A20 harp is capable of directly measuring beam profiles in the AGS as delivered from the Booster and through the BTA beamline. The A20 harp is also effective in capturing profiles of consecutive turns about the AGS. Vertical steering magnet DV120, located in the BTA line, was used to mis-steer the beam. The beam current was monitored during this operation to assure minimal beam loss due to the beam mis-steering. The AGS vertical nunemeter kicker was timed to drive the beam into a beam scraper after the desired number of turns. The beam profile of injection turn, after one-turn revolution and after two-turn revolution were obtained by turning on the kicker in the first three consecutive turns. These three profiles are shown in Fig. 3. By fitting these three beam profiles to separated Gaussian distributions, the beam widths of these three consecutive tums were given as $\sigma_{0}=4.4 \mathrm{~mm}, \sigma_{1}=5.0 \mathrm{~mm}, \sigma_{2}=5.2 \mathrm{~mm}$, respectively.

At the given position of the lattice, the beam profile is determined by three parameters: $\epsilon, \alpha, \beta$. The AGS ring parameters $\alpha, \beta$ and betatron tunes are known fairly well, therefore a one turn transfer matrix can be easily generated. These three parameters of the beam can be solved using the width measurements in three turns, therefore determining the three unknowns $\epsilon, \alpha$ and $\beta$ of the incoming beam.

Assuming the emittance does not change in the first three 
turns, by definition,

$$
\epsilon=\pi \frac{\sigma_{0}^{2}}{\beta_{0}}=\pi \frac{\sigma_{1}^{2}}{\beta_{1}}=\pi \frac{\sigma_{2}^{2}}{\beta_{2}},
$$

where $\sigma$ is the beam width, $\beta$ is the beta functions of the beam, and subscripts 0,1 , and 2 stand for injection turn, first turn and second turn, respectively. The single particle emittance can be written in the phase space coordinates $y, y^{\prime}$,

$$
\begin{aligned}
\epsilon & =\gamma_{0} y_{0}^{2}+2 \alpha_{0} y_{0} y_{0}^{\prime}+\beta_{0} y_{0}^{\prime 2} \\
& =\gamma_{1} y_{1}^{2}+2 \alpha_{1} y_{1} y_{1}^{\prime}+\beta_{1} y_{1}^{\prime 2}, \\
& =\gamma_{2} y_{2}^{2}+2 \alpha_{2} y_{2} y_{2}^{\prime}+\beta_{2} y_{2}^{\prime 2}
\end{aligned}
$$

where $y_{0}, y_{0}^{\prime} ; y_{1}, y_{1}^{\prime}$ and $y_{2}, y_{2}^{\prime}$ are phase space coordinates of the injection tum, the first turn and the second turn, respectively. Using a one turn mapping matrix $M$, we have

$$
\left(\begin{array}{l}
y_{1} \\
y_{1}^{\prime}
\end{array}\right)=M\left(\begin{array}{l}
y_{0} \\
y_{0}^{\prime}
\end{array}\right)=\left(\begin{array}{ll}
C_{1} & S_{1} \\
C_{1}^{\prime} & S_{1}^{\prime}
\end{array}\right)\left(\begin{array}{l}
y_{0} \\
y_{0}^{\prime}
\end{array}\right),
$$

with

$$
M=\left(\begin{array}{cc}
\cos 2 \pi \nu+\alpha_{r} \sin 2 \pi \nu & \beta_{r} \sin 2 \pi \nu \\
-\gamma_{r} \sin 2 \pi \nu & \cos 2 \pi \nu-\alpha_{r} \sin 2 \pi \nu
\end{array}\right)
$$

where the subscript $r$ corresponds to the parameters at the harp location in the AGS ring. These parameters can be determined fairly well using MAD calculation. Similarly,

$$
\left(\begin{array}{l}
y_{2} \\
y_{2}^{\prime}
\end{array}\right)=M\left(\begin{array}{l}
y_{1} \\
y_{1}^{\prime}
\end{array}\right)=\left(\begin{array}{ll}
C_{1} & S_{1} \\
C_{1}^{\prime} & S_{1}^{\prime}
\end{array}\right)\left(\begin{array}{l}
y_{1} \\
y_{1}^{\prime}
\end{array}\right) .
$$

After some algebra, the injected beam parameters can be written as

$$
\begin{aligned}
\beta_{0} & =\frac{1}{\sqrt{\left(\frac{\sigma_{1}^{2}}{\sigma_{0}^{2}}\right)\left(\frac{1}{S_{1}^{2}}\right)-\left(\frac{C_{1}}{S_{2}}\right)^{2}+\frac{C_{1}}{S_{1}} A-\frac{1}{4} A^{2}}}, \\
\alpha_{0} & =\frac{1}{2} \beta_{0} A \\
\epsilon_{0} & =\pi \frac{\sigma_{0}^{2}}{\beta_{0}},
\end{aligned}
$$

where

$$
A=\frac{\left(\frac{\sigma_{1}}{\sigma_{0}}\right)^{2} \frac{1}{S_{1}^{2}}-\left(\frac{\sigma_{2}}{\sigma_{0}}\right)^{2} \frac{1}{S_{2}^{2}}-\left(\frac{C_{1}}{S_{1}}\right)^{2}+\left(\frac{C_{2}}{S_{2}}\right)^{2}}{\left(\frac{C_{2}}{S_{2}}\right)-\left(\frac{C_{1}}{S_{1}}\right)} .
$$

With measured betatron tune and beam widths $\sigma_{0}, \sigma_{1}$ and $\sigma_{2}$, the injected beam parameters $\alpha, \beta$ and $\epsilon$ can be determined uniquely. The vertical betatron tune was measured as 8.66. The ring parameters at the multi-wire location as calculated using MAD are $\alpha_{r}=-1.25 ; \beta_{r}=$ $14.25 \mathrm{~m}$. Substituting the beam size values

$$
\sigma_{0}=4.4 \mathrm{~mm}, \sigma_{1}=5.0 \mathrm{~mm}, \sigma_{2}=5.2 \mathrm{~mm}
$$

into Eq.(4), the injected beam parameters are

$$
\alpha_{0}=-1.09 ; \beta_{0}=11.95 \mathrm{~m}, \epsilon_{0}=1.67 \pi \mathrm{mm}-\mathrm{mrad} .
$$

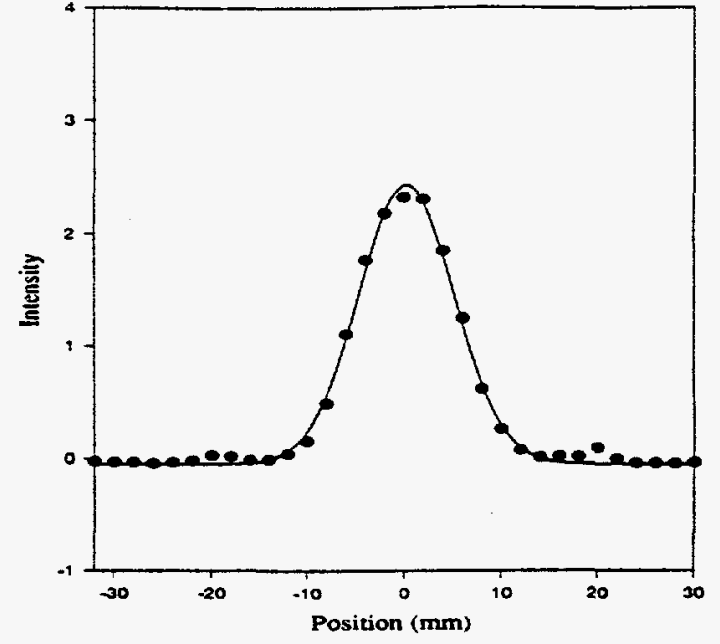

Figure 4: The multi-turn vertical beam profile taken by the A20 harp. Solid points are the experimental data and the solid curve is Gaussian fit. Low sensitivity gain was used.

Thus the normalized $95 \%$ vertical beam emittance is $\epsilon_{95 \%}^{v}=6 \beta \gamma \epsilon_{0}=4.3 \pi \mathrm{mm}$-mrad. The measured emittance at the injection of the AGS using IPM is $5 \pi \mathrm{mm}$-mrad. Further experiment is needed to understand the emittance growth mechanism.

A multi-turn profile measurement was also performed without mis-steering of DV120, resulting in the diluted vertical beam profile. Beam loss associated with the scattering between the beam and multi-wire monitor limits beam survival to less than 100 rums. A profile is shown in Fig. 4 where the beam width is $\sigma_{0}=5.5 \mathrm{~mm}$ and the corresponding beam emittance is $\epsilon_{95 \%}^{v}=5.3 \pi \mathrm{mm}$-mrad, which is slightly larger than the value measured by the AGS IPM and is due to the scattering between the beam and the wires.

\section{CONCLUSION}

A multi-wire beam profile monitor has been installed and tested in the Brookhaven AGS. It can be used to directly measure the beam profile being delivered from the Booster through the BTA beamline, and the evolution of this profile in consecutive turns about the AGS. The system can also be used to simply tune injection, if an optical match to the AGS lattice is desired. As the AGS prepares to serve as the injector for RHIC, it will justify its importance in transverse emittance control.

\section{REFERENCES}

[1] H. Weisberg, et al., Proc. IEEE Particle Accelerator Conference, 1983, p. 2179 .

[2] R.L. Witkover, et al., Proc. IEEE Particle Accelerator Conference, 1987, p. 567 .

[3] G.A. Smith, et al., Proc. IEEE Particle Accelerator Conference, 1991 , p.1264. 


\section{DISCLAIMER}

This report was prepared as an account of work sponsored by an agency of the United States Government. Neither the United States Government nor any agency thereof, nor any of their employees, makes any warranty, express or implied, or assumes any legal liability or responsibility for the accuracy, completeness, or usefulness of any information, apparatus, product, or process disclosed, or represents that its use would not infringe privately owned rights. Reference herein to any specific commercial product, process, or service by trade name, trademark, manufacturer, or otherwise does not necessarily constitute or imply its endorsement, recommendation, or favoring by the United States Government or any agency thereof. The views and opinions of authors expressed herein do not necessarily state or reflect those of the United States Government or any agency thereof. 


\section{DISCLAMMER}

Portions of this document may be illegible in electronic image products. Images are produced from the best available original document. 